\title{
Philosophiques
}

\section{Julia Kristeva, Le Génie féminin. Hannah Arendt, Paris, Fayard, 1999, 408 pages.}

\section{Luc Vigneault}

Volume 30, numéro 2, automne 2003

URI : https://id.erudit.org/iderudit/008658ar

DOI : https://doi.org/10.7202/008658ar

Aller au sommaire du numéro

Éditeur(s)

Société de philosophie du Québec

ISSN

0316-2923 (imprimé)

1492-1391 (numérique)

Découvrir la revue

Citer ce compte rendu

Vigneault, L. (2003). Compte rendu de [Julia Kristeva, Le Génie féminin. Hannah Arendt, Paris, Fayard, 1999, 408 pages.] Philosophiques, 30(2), 461-465.

https://doi.org/10.7202/008658ar d'utilisation que vous pouvez consulter en ligne.

https://apropos.erudit.org/fr/usagers/politique-dutilisation/ 
propositions de Chauvier ne sont-elles pas équivalentes à celles de Rawls? De mon point vue, cela fait peu de doute: le principe de socialisation des avantages proposé par Chauvier à la fin de son ouvrage est tout à fait analogue au "devoir d'assistance» proposé par Rawls dans The Law of Peoples (Cambridge, Mass., Harvard University Press, 1999). Dans le cas contraire, à supposer que les propositions substantielles du philosophe français soient nettement novatrices par rapport à celles de Rawls, alors Chauvier devra nous expliquer pourquoi il s'est fait le défenseur des idées de Rawls dans un récent article ("Les principes de la justice distributive sont-ils applicables aux nations?", Revue de métaphysique et de morale, $\mathrm{n}^{\circ}$ 1, 2002, p. 123-143).

Ainsi se pourrait-il que cet ouvrage en fasse à la fois trop et pas assez, c'està-dire qu'il critiquerait trop pour le peu qu'il aurait finalement à proposer? Il reste que Justice internationale et solidarité pose d'importantes questions et qu'il initie le lecteur francophone aux débats les plus importants de l'heure en théorie de la justice internationale.

MARTIN LEBLANC Université de Montréal

\section{Julia Kristeva, Le Génie féminin. Hannah Arendt, Paris, Fayard, 1999, 408 pages.}

Linguiste, romancière, archéologue du langage, Julia Kristeva arpente depuis plus de trente ans les vastes palais du langage et l'univers symbolique humain sous différentes formes. On lui doit plusieurs ouvrages majeurs dans le domaine littéraire, sémiologique et linguistique, mais la grande originalité de l'œuvre de Kristeva est sans doute la contribution psychanalytique qu'elle apporte au domaine du langage et, ce faisant, la contribution qu'elle apporte au développement de la psychanalyse par ses analyses du langage. La folie, le langage, la révolte, l'amour et le féminin demeurent ses thèmes charnières. Ceci demeure vrai de son dernier ouvrage, une trilogie centrée sur le thème du "génie féminin ». Trois lieux définiront ce "génie du féminin»: la vie, la folie et les mots. Trois auteures correspondent à ces trois lieux, les "plus sensibles" du Xx siècle: Hannah Arendt, Mélanie Klein et Colette. Je présente ici le premier des tomes sur Hannah Arendt ainsi qu'un résumé de la trilogie telle que Kristeva l'annonce dans l'introduction de son livre.

D'un ton prophétique, Kristeva annonce une ère du féminin à venir: "le siècle prochain sera féminin pour le meilleur ou pour le pire" (p. 11). Le "génie", cette extraordinaire faculté qu'on accordait jadis à qui étaient bénis des dieux, s'est aujourd'hui mué en la capacité particulière à innover. On comprendra ainsi que la triologie commence avec Hannah Arendt, qui a fait de cette aptitude le centre de sa théorie de l'action. Le génie est celui qui sait innover; plus particulièrement, le génie caractérise la personne singulière dont les expériences propres, les "excès surprenants» de l'existence surgissent au-delà d'un univers de plus en plus standardisé (p. 8). Historiquement, les femmes semblent avoir été rejetées de cette catégorie, en vertu de leur don spécifique de maternité. L'expérience possible d'une "osmose avec l'espèce» différencie radicalement le genre féminin des hommes, mais cette différence justement s'ajoute aux nombreuses difficultés à manifester 
leur génie (p. 13). Indéniablement, les mères peuvent être des génies naturels de l'amour et du dévouement, mais, plus encore, une certaine manière de vivre la vie de l'esprit leur confère un "génie bien à elles" (p. 14).

C'est ce caractère binaire du génie féminin, combinant pensée et expérience, raison et sensibilité, qui orientera le projet de Kristeva. Deux angles de lecture se superposent avec persistance: un angle plus conceptuel présentant les grands thèmes de la pensée arendtienne, et un angle psychologique où Kristeva se livre généreusement à l'analyse des souffrances psychiques du personnage "Arendt". Trois traits dominants forment la constellation de ce personnage: sa condition de femme, sa judaïté et sa libre pensée. Si Arendt n'a jamais assumé pleinement ni sa condition proprement féminine, ni sa judaïté, elle les a admirablement sublimées dans la vigueur (il faudrait dire la virilité) peu commune de sa libre pensée. Au surplus, poursuit Kristeva, Arendt ne semble avoir assumé ni son corps et sa sexualité, ni la douleur causée par la mort de son père et transférée dans sa liaison avec Heidegger, lequel incarnera la vraie figure du père et de l'autorité à dépasser. Selon Kristeva, Arendt avait donc tout un attirail de mécanismes de défense, et sa singulière et bien connue aversion pour la psychanalyse ou toute forme de "psychologie des profondeurs ", qu'elle associait au culte moderne de la personnalité, n'en aura été que la plus manifeste des confirmations. Son génie, on se le demandera sans cesse au cours de la lecture de l'ouvrage, n'aurait-il été que celui de sublimer, mieux que les autres, ses propres refoulements?

Selon Kristeva, c'est autour du concept de vie, non comme zoè, vie naturelle et biologique, mais comme bios, vie spécifiquement humaine, que se concentre l'œuvre d'Arendt. Plus précisément, la tension entre la zoè et le bios forme le creuset où se joue le destin de la vie humaine singulière. La vie sous toutes ses formes, de la natalité à la vie de l'esprit, est le bien le plus précieux et devient la valeur ultime à préserver devant l'effondrement des principes qui guidaient notre modernité et que les horreurs totalitaires ont fait sombrer dans une nuit sans fond. Au contraire, et en débat constant avec son maître et amant de jeunesse, Heidegger, Arendt n'a jamais sacrifié la singularité de la vie à la tâche de la pensée; elle a plutôt défendu l'idée que la philosophie, même en vertu de son retrait nécessaire hors du monde, doit demeurer engagée dans la vie du sens commun. Telle est la spécificité du "génie" d'Arendt: vivre sa pensée, vivre de ses inventions "versées au développement de la pensée et des êtres, de la floraison de questions ». Tel est le génie d'Arendt: «une œuvre enracinée dans la biographie de l'expérience » et "qui nous oblige à raconter son histoire» (p. 9).

Trois chapitres donnent le rythme de l'ouvrage sur Arendt; trois chapitres qui respectent sensiblement l'itinéraire d'Arendt. Le premier chapitre couvre la période de jeunesse jusqu'à son exil aux États-Unis (1906-1940) et constitue la partie la plus longue du livre. Le second chapitre se centre autour de la période de la maturation de sa théorie politique (1941-1970) et aborde principalement deux questions: la question juive et le totalitarisme. Le dernier chapitre sera entièrement consacré à un débat avec la dernière œuvre d'Arendt: la trilogie de La Vie de l'esprit (1971-1975).

Le titre du premier chapitre, "La vie est un récit», annonce les intentions de Kristeva : situer le projet arendtien comme une apologie de la narration. Mais c'est aussi le récit de la vie d'Arendt que mène Kristeva, récit où s'entremêlent description biographique et analyse du personnage "Arendt». Pourquoi cette 
distinction? La thèse d'Arendt sur Le Concept d'amour chez Augustin devient, pardelà l'enjeu théorique, un véritable débat personnel avec sa propre souffrance vécue dans l'échec de la relation passionnelle avec Heidegger. La thèse sur Augustin ne devient-elle pas l'occasion d'une activité compensatoire ou, mieux, peut-être, d'une sublimation? Son destin se trace entre la tension de la zoè et du bios, entre la pulsion brute de la naturalité et la sublimation suprême qu'est l'exercice de sa pensée. L'enjeu de la tension entre naturalité (subir ses pulsions inconscientes) et sa sublimation se joue vraisemblablement dans la figure de l'amour dont le paradigme est l'amour maternel. Cette sublimation constituerait la véritable vie humaine, une vie comme récit, car elle seule témoigne d'une maternité affranchie, c'est-à-dire ayant assumé la tension amoureuse de la zoè et du bios vers un amour désintéressé. Cet amour désintéressé, Kristeva le nomme, reprenant l'idée chère à Arendt d'un amor mundi, l'amour pour le quelconque. Amour maternel, amour pour le prochain, pour l'étranger-au-monde, «tout aussi fragile que moi devant la mort et qui par mon amour de femme mère, réinvente continuellement le sens infini de vies plurielles dont il me fait don en retour ", l'éloge du concept de natalité arendtien.

C'est encore ce destin de la pulsion amoureuse qui se retrouve dans l'analyse de la biographie sur Rahel Varnhagen qu'Arendt rédige avant son départ définitif de l'Allemagne en 1933. Si les travaux sur Augustin sont l'occasion d'une élucidation de cette pulsion, la confrontation entre «Rahel» et «Hannah» a pour enjeu la judéo-féminité d'Arendt. Cette biographie, pour Kristeva, est bien une «autoanalyse » qui consiste à «traverser l'hystérie de cette femme, non pas pour l'évacuer, ni même pour l'analyser, mais pour la transformer (sublimer) en "vivre bien" (eudzèn), selon l'idéal d'Aristote » (p. 119). Tâche castratrice dont Arendt se tire fort bien. Sa vie peut ainsi s'inscrire comme récit et dépasser la voie de la pure répétition. C'est sur ce terrain de l'expérience qu'Arendt peut tisser sa propre théorie de la narration; théorie qu'elle affine dans sa lecture d'Aristote. L'interprétation que fait Kristeva sur ce rapport entre Arendt et Aristote est sans aucun doute le moment fort de ce chapitre, car cette interprétation demeure auprès du penser arendtien. Arendt trouve effectivement chez Aristote la faculté mentale politique par excellence dans le concept de phronêsis, cette faculté qui, opposée à la sophia de Platon, épouse la condition humaine de la praxis. La phronêsis, faculté au savoir limité, mais qui en tant que sagesse connaît précisément ses limites, demeure auprès du monde phénoménal et a pour objet "non seulement les universels, mais aussi les singuliers, car elle est de l'ordre de l'action» (p. 134). Cette lecture d'Aristote, Kristeva en convient, est un moment clé de la pensée arendtienne, car c'est cette même aptitude à juger les événements singuliers qu'Arendt cherchera à thématiser, beaucoup plus tard, dans l'analyse politique du jugement esthétique kantien.

La perspicacité jugeante, parce qu'elle est faite pour l'action, est aussi faite pour l'exercice d'un récit. Arendt en cherchera une praxis dans l' «art de raconter» le roman du siècle: Les Origines $d u$ totalitarisme. Loin d'être une analyse théorique minutieuse de cet ouvrage monumental, Kristeva se contente, dans la seconde partie de son étude, de retracer les grandes lignes de la thèse arendtienne. Elle cherche à retracer, comme elle le fera pour les autres thèmes, les conditions d'émergence (l'anamnèse psychanalytique) de la théorie sur le totalitarisme. La préoccupation centrale de ce chef-d'œuvre est de retracer la cristallisation d'un mal absolu qui a pour effet de détruire la spécificité humaine de la pluralité. Au nom de quoi peuton décider que tel ou tel individu, tel ou tel groupe d'individus n'ont pas le droit 
de vivre sur cette terre? Aucun concept ne peut justifier l'horreur, sinon précisément l'absence de fondement qui caractérise l'entreprise totalitaire. Ce deuxième chapitre sera bonifié par quelques allusions à l'Essai sur la révolution et au cas Eichmann.

Le grand absent de ce chapitre, et de l'étude de Kristeva en général, est sans doute le maître ouvrage Condition de l'homme moderne. Hormis quelques pages dans la dernière section du second chapitre, il n'y a aucune analyse de cette œuvre. De la même façon, l'action, l'activité politique par excellence pour Arendt, ne fait l'objet d'aucun traitement approfondi. Peut-être finalement que cette œuvre d'Arendt, de fait, ne se prête pas aussi facilement à un traitement psychanalytique et qu'on aurait de toute façon peu à tirer d'un tel exercice.

Le troisième et dernier chapitre comble en partie ce vide en revenant sur la question de l'identité. Traitant essentiellement de La Vie de l'esprit, la dernière partie de l'ouvrage de Kristeva, malgré quelques ambiguïtés, dépasse de loin le reste et constitue une analyse en soi de la pensée arendtienne. Retournant au dualisme initial zoè-bios, Kristeva introduit la problématique nettement déconstructiviste de La Vie de l'esprit par la dialectique du qui et du corps. Le qui, nom que donne Arendt à l'identité politique, est précisément un arrachement à la vie naturelle, " une vie biologique dont l'être humain doit s'extraire pour conquérir sa spécificité » (p. 277). Le qui n'est pas l'identité au sens de la représentation de soi, mais l'identité de la vie de quelqu'un. Cette identité narrative qui ne peut être saisie par le sujet lui-même "se révèle uniquement dans l'histoire de sa vie, c'est-à-dire lorsque la vie s'en va, ne laissant derrière elle qu'une histoire" (Condition de l'homme moderne, p. 252). Elle correspond à ce que Platon appelait le daîmon, cette identité qui «n'apparaît et n'est visible qu'aux autres". L'action n'a donc de sens que dans l'horizon de cette identité. Cette identité est celle du bios. Le grand oublié de cette question identitaire, selon Kristeva, c'est le corps qui fut en réalité non pas oublié, mais occulté radicalement. Le corps n'est pas un concept dans l'ordre du général. Irréductiblement mentaliste, Arendt aurait fait du corps, comme de l'amour, un «objet de pensée ». Elle occulte en ce sens la faculté d' "avoir du plaisir », dimension qui pourtant se retrouve au centre de la théorie arendtienne du jugement, mais qui ne serait pas exploitée en raison des résistances d'Arendt à aborder cette question.

Analyse plus pénétrante, selon Kristeva, la problématisation du conflit entre le «Moi pensant» et le "Moi » permet à Arendt d'affiner sa critique des paradigmes fallacieux de la métaphysique (p. 312). Arendt insiste ici sur la conflictualité de l'expérience de la pensée. Dans ce conflit du Moi pensant et du Moi, la pensée se dédouble, et dans ce dédoublement, temporel et spatial, la pensée privilégie le Moi pensant. Ce lieu de la pensée n'est pourtant pas le lieu de l'esseulement, car dans la pensée, "l'homme se tient compagnie à lui-même», ce qui veut dire qu'il maintient le lien d'amitié avec le sens commun qu'il partage et qui s'insinue dans le dialogue sous l'aspect du Moi opposé au Moi pensant. Le «penchant tyrannique» de la philosophie ne vient-il pas de l' "écart entre pensée et monde » et, plus encore, de l' "interdépendance des deux» (p. 313) ? La pensée est-elle en mesure d'assumer sa propre «psychose», sa «dualité originelle», sa propre faille? s'interroge Kristeva. "Délicieuse cicatrice» de ce dédoublement originaire, de la «suture ", la pensée, dans sa dangerosité pour l'équilibre politique (en raison de son incessante remise en question), recèle aussi ses propres ressources stabilisatrices: le dialogue et l'amitié nécessaire à son actualisation, pour autant qu'elle ne verse pas dans ses «latences paranoïdes» qui font de la maîtrise de son étonnement systématisé une 
volonté de "maîtriser le monde à son tour» (p. 314). La pensée se ferait-elle volonté de puissance ? La volonté, comme le prétend Kristeva, ne serait-elle qu'une modalité de la pensée, à l'instar du jugement? Une ambiguïté persiste dans ce passage de la pensée à la volonté et, plus tard, de la pensée au jugement. Il semble pourtant qu'Arendt fait de ces trois activités solidaires, comme elle le fait avec les trois activités de la vita activa, des activités distinctes de la vie de l'esprit. Peut-être qu'une analyse plus serrée du conflit entre le Moi-voulant et le Moi-pensant, que Kristeva n'aborde pas, aurait dissipé cette ambiguïté. L'analyse de la volonté demeure axée sur la lecture arendtienne des penseurs du christianisme. Retour en force à sa lecture d'Augustin et au pouvoir de commencer, la pensée chrétienne permet à Arendt de rouvrir la thématique du commencement et de la fonder dans une philosophie de la volonté. Le commencement chrétien, écrit Kristeva, «apparaît ainsi comme un commencement de la volonté qui ouvre la problématique de la liberté et comporte deux conséquences majeures: il creuse l'espace de l'homme intérieur, amorcé par les stoïciens, et s'achève en une volonté de puissance, qui se révèle une volonté de vie» (p. 319).

Bien qu'elle reproche à Arendt, avec raison, sa réduction de l'importance stoïcienne dans le concept d'un espace mental intérieur et son esquive, encore une fois, d'un sujet hautement psychologique, Kristeva salue l'initiative d'Arendt de raccrocher la volonté au projet d'une «humanité naissancielle» (p. 331). C'est encore cette faculté de volonté, qu'elle trouve de façon paradigmatique chez Duns Scot, le seul des penseurs de la volonté «à payer le prix de la contingence en échange du don de liberté»(p. 340), qui lui permet d'étayer sa critique contre les "philosophes professionnels » qui persistent à s'enfermer dans une liberté philosophique, laissant pour compte la liberté politique. Théoricienne du «fait de la natalité ", Arendt cherche dans les conditions de la vie humaine la possibilité de recommencer quelque chose de neuf sans pour autant renier ce qui s'est passé. S'inspirant de Kant, Arendt cherchera à travers une thématisation du jugement à cerner une faculté proprement politique de l'esprit, faculté de l'esprit qui atteste l'appartenance immédiate des êtres humains à la communauté. En terminant son ouvrage sur les thèmes de la promesse et du pardon, deux véritables "mécanismes régulateurs " de la faculté de juger, Kristeva revient sur ce qui semble être le cœur de la pensée arendtienne: non pas l'élaboration d'une nouvelle théorie de la liberté politique, mais le pari politique que les hommes, en exerçant leur jugement, se révèlent des êtres "naissanciels ", toujours capables du plus imprévisible. C'est au cœur de cette expérience totale de la natalité que s'ancre la capacité thaumaturgique des hommes, capables non seulement de faire des miracles, mais des miracles «qui sauvent le monde» (p. 371).

En se rabattant finalement sur ces deux mécanismes régulateurs du jugement, Kristeva nous permet de mieux saisir la cohérence de la méditation arendtienne de la condition humaine. Par contre, si la grande contribution de cet ouvrage repose à mon sens sur l'audace d'aborder, dans une perspective narrative, la trilogie de $L a$ Vie de l'esprit, on n'y trouve aucune élucidation plus explicite de la place du jugement au sein des activités de l'esprit. Le lecteur habitué aux textes arendtiens n'y trouvera rien qui permette une nouvelle interprétation de cette triologie, mais peut-être une nouvelle manière de voir ce qu'a vraiment été le génie de cette femme. 